\title{
Dissipative Control of Energy Flow in Interconnected Systems
}

\author{
Y. Kishimoto ${ }^{1}$ and D. S. Bernstein ${ }^{2}$ \\ University of Michigan, Ann Arbor, MI \\ S. R. Hall ${ }^{3}$ \\ Massachusetts Institute of Technology, Cambridge, MA
}

\begin{abstract}
Abstruct
Dissipative energy flow controllers are designed for interconnected modal subsystems. Active feedback controllers for vibration suppression are then viewed as either an additional subsystem or a dissipative coupling. These controllers, which are designed by the LQG positive real control approach, maximize energy flow from a specified modal subsystem.
\end{abstract}

\section{Introduction}

Energy flow has been widely studied as an effective tool for analyzing large, interconnected vibrating systems. One of the key results of this approach is the fact that, within interconnected subsystems, energy flow can often be expressed as a linear combination of subsystem energy.

In active control for reducing vibration, energy flow has been considered as a performance index to be minimized [1]-[6]. The design of these active controllers, however, has some difficulties. For example, the optimal controller is often noncausal [5] and thus asymptotic stability of the closed-loop system cannot be guaranteed. Furthermore, active energy flow control for interconnected structures composed of more than two subsystems has received limited attention due to the lack of energy flow models for such interconnected systems.

In recent work $[7,8]$ motivated by Wyatt et al. $[9]$ an energy flow model was derived for a structure consisting of several modal subsystems that are coupled either conservatively or dissipatively. In the present paper, our goal is to design active control laws for coupled subsystems by applying these energy flow models.

Three typical situations requiring energy flow controllers are considered in this paper. First, in Section 4, we consider energy flow control for several subsystems interconnected by conservative coupling [7]. For such an interconnected system, the control law can be designed for the system as a whole, which requires an energy flow model for the entire system including the controller. We thus treat the controller as an additional subsystem interconnected by a conservative coupling, so that energy flow is controlled through the coupling.

Next, in Section 5, we consider energy flow control among individual structural modes. Ilere we exploit the fact that structural modes are essentially coupled by the input and output matrices. By appropriately enlarging the input and output matrices, we design a dissipative controller that serves, in effect, as a dissipative coupling [8]. As an application of this approach, in Section 6 we consider two uncoupled systems that are controlled by a relative force.

${ }^{1}$ Graduate Student, Department of Aerospace Engineering

${ }^{2}$ Associate Professor, Department of Aerospace Engineering

${ }^{3}$ Associate Professor, Department of Aeronantics and Astronautics

Senior Member AIAA

Copyright (C)1993 by Y. Kishimoto, D.S. Bernstein, S.R. Hall.

Published by the American Institute of Aeronautics and Astronautics, Inc., with permission.
In both cases the controller is designed to maximize the steady state energy flow from one of the subsystems, so that we can reduce the vibration of the specified subsystem. The control approach we use is due to Lozano-Leal and Joshi [10]. This approach is briefly reviewed in Section 3. Since the controller and plant are both positive real, closed-loop asymptotic stability is guaranteed in spite of modeling uncertainty.

\section{Energy Flow Model for Interconnected Systems}

In this section we briefly review some results concerning energy flow obtained in [7]-[9]. Consider $r$ subsystems $z_{1}(s) \ldots$, $z_{r}(s)$ interconnected by a linear time-invariant coupling $L(s)$. Each subsystem $z_{i}(s)$ is assumed to be a strictly positive real and thus asymptotically stable scalar transfer function. Now we define normalized white noise $\tilde{w}(t) \triangleq\left[\tilde{w}_{1}(t) \cdots \bar{w}_{n}(t)\right]^{\mathrm{T}}$ whose intensity matrix is identity. Assume that the disturbance vector $w_{0}(t) \triangleq\left[w_{1}(t) \cdots u_{r}(t)\right]^{\mathrm{T}}$ is now given by

$$
w_{0}(t)=D \tilde{w}(t)
$$

where $D \in \mathcal{R}^{r \times n}$ is the constant matrix, then the intensity matrix $S_{u_{0} w_{0}}$ of $w_{0}(t)$ is given by $S_{w_{0} w_{0}}=D D^{\mathbf{T}}$.

For later use, define $r \times r$ diagonal transfer function

$$
Z(s) \triangleq \operatorname{diag}\left(z_{1}(s), z_{2}(s), \ldots, z_{T}(s)\right) .
$$

By defining the $r$-dimensional vectors

$$
u_{0} \triangleq\left[u_{1} \cdots u_{r}\right]^{\mathrm{T}}, y_{0} \triangleq\left[y_{1} \cdots y_{r}\right]^{\mathrm{T}}, v_{0} \triangleq\left[v_{1} \cdots v_{r}\right]^{\mathrm{T}},
$$

we obtain the feedback representation of the interconnected system shown in Fig. 2.1 in terms of $Z^{-1}(s)$, which is strictly positive real, and where $v_{0}=L y_{0}$ and $u_{0}=w_{0}-v_{0}$.

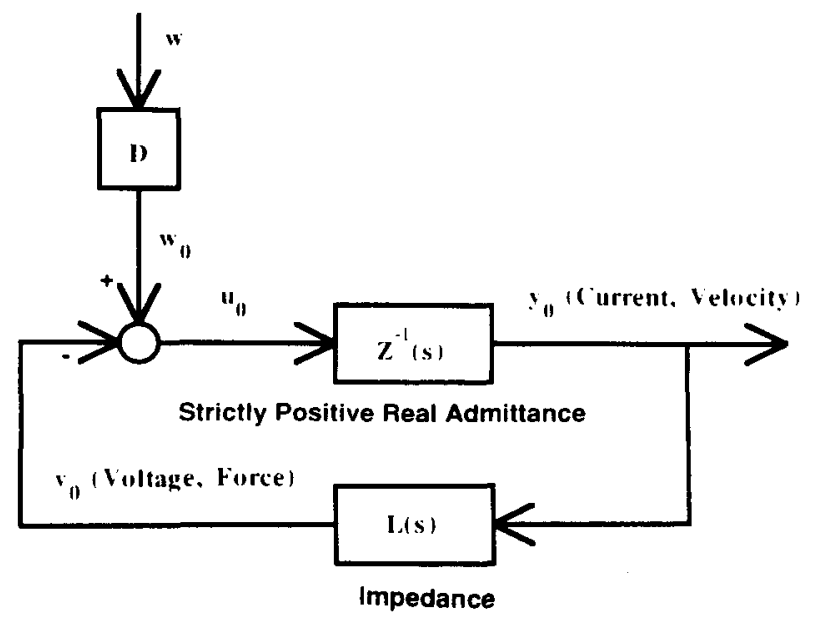

Fig. 2.1. Feedhack Representation of Interconnerted Systcm. 
We now introduce three stcady state energy flows $P_{i}^{\mathrm{P}}, P_{i}^{4}$, $P_{i}^{\prime \prime}, i=1, \ldots, r$,

$P_{i}^{\mathrm{p}}=$ the steady state average encrgy flow entering the $i$ th subsystem throught the $i$ th port of $L(s)$,

$P_{i}^{d}=$ the stearly state average energy dissipation rate of the $i$ th sulssystem,

$P_{i}^{e}=$ the steady state average external energy flow entering the $i$ th subsystem.

Fig. 2.2 illustrates 1he resulting energy flow model for the case $r=3$.

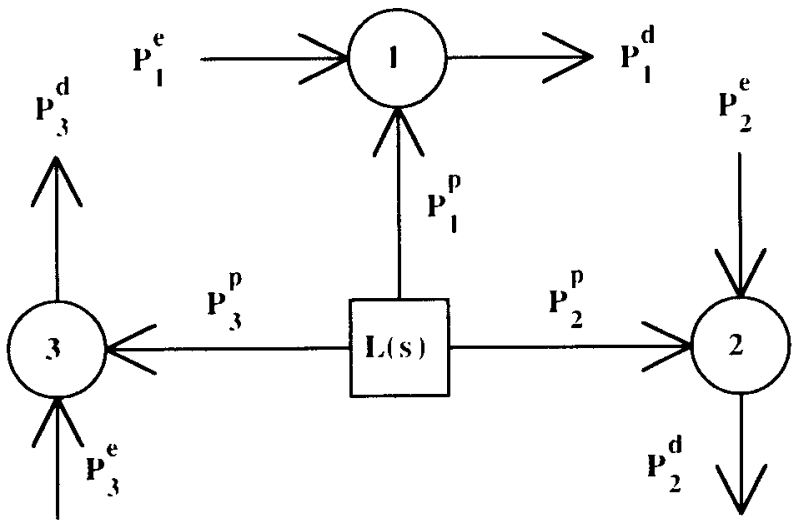

Fig. 2.2. Energy Flow Model with Three Subsystoms.

Furthermore, these encrgy flows satisfy the conservation of energy, that is,

$$
P_{i}^{\mathrm{p}}+P_{i}^{\mathrm{d}}+P_{i}^{t}=0, \quad i=1, \ldots, r .
$$

\section{LQG Positive Real Control Approach}

In this section we briefly review the $L Q G$ positive real control approach developed in [10].

The LQG control approarh provides the optimal controller for the following problem. Given the $n$ th-order stabilizalle and detectable plant

$$
\begin{gathered}
\dot{x}(t)=A x(t)+B u(t)+D_{1} \tilde{w}(t), \\
y(t)=C x(t)+D_{2} \tilde{w}(t),
\end{gathered}
$$

determine an $n$ tl 1 -order dynamic feedback compensator $G_{c}(s) \sim$ $\left[\begin{array}{c|c}A_{\mathrm{c}} & B_{\mathrm{c}} \\ \hline C_{\mathrm{c}} & 0\end{array}\right]$ of the form

$$
\begin{gathered}
\dot{x}_{\mathrm{c}}(t)=A_{c} x_{\mathrm{c}}(t)+B_{\mathrm{c}} y(t), \\
u(t)=C_{\mathrm{c}} x_{\mathrm{c}}(t),
\end{gathered}
$$

such that the closed-loop system (4) - (7) with dynamics matrix

$$
\tilde{A} \triangleq\left[\begin{array}{cc}
A & B C_{c}^{\prime} \\
B_{c} C & A_{\mathrm{c}}
\end{array}\right]
$$

is asymptotically stable and the $I_{2}$ performance index

$$
\begin{aligned}
J\left(A_{\mathrm{c}}, B_{c}, C_{\mathrm{c}}\right) & =\lim _{t \rightarrow \infty} \mathcal{E}\left\{\frac{1}{t} \int_{0}^{t}\left[x^{\mathrm{T}}(s) R_{1} x(s)+u^{\mathrm{T}}(s) R_{2} u(s)\right] d s\right. \\
& =\|\tilde{G}(s)\|_{2}^{2}
\end{aligned}
$$

is minimized, where

$$
\tilde{G}(s) \sim\left[\begin{array}{c|c}
\tilde{A} & \tilde{D} \\
\hline \tilde{E} & 0
\end{array}\right]
$$

is the closed-loop transfer fuuction from the unit intensity white noise disturbance $\tilde{w}(t)$ to the performance variables $z(t)=E_{1} x(t)+$ $E_{2} u(t)$ and where $\tilde{D} \triangleq\left[\begin{array}{c}D_{1} \\ B_{c} D_{2}\end{array}\right], \tilde{E} \triangleq\left[\begin{array}{ll}E_{1} & E_{2} C_{\mathrm{c}}\end{array}\right], R_{1} \triangleq$ $E_{1}^{\mathrm{T}} E_{1}, R_{2} \triangleq E_{2}^{\mathrm{T}} E_{2}>0$ and $E_{1}^{\mathrm{T}} E_{2}=0$. It is assumed that $A, B, C, D_{1}$ and $E_{1}$ satisfy $(i)(A, B)$ and $\left(A, D_{1}\right)$ are stabilizable, and (ii) $\left(C^{\prime}, A\right)$ and $\left(E_{1}, A\right)$ are detectable. Furthermore, for convenience, define $V_{1} \triangleq D_{1} D_{1}^{\mathrm{T}}, V_{2} \triangleq D_{2} D_{2}^{\mathrm{T}}>0$, and assume that $D_{1} D_{2}^{\mathrm{T}}=0$.

For this problem the optimal compensator $\left(A_{c}, B_{c}, C_{c}\right)$ is given by

$$
\begin{gathered}
A_{c}=A-Q C^{\mathrm{T}} V_{2}^{-1} C^{\prime}-B R_{2}^{-1} B^{\mathrm{T}} P \\
B_{c}=Q C^{\mathrm{T}} V_{2}^{-1} \\
C_{c}=-R_{2}^{-1} B^{\mathrm{T}} P
\end{gathered}
$$

where $Q$ and $P$ are $n \times n$ nonnegative-definite matrices satisfying

$$
\begin{aligned}
& A Q+Q A^{\mathrm{T}}+V_{1}-Q C^{\mathrm{T}} V_{2}^{-1} C Q=0, \\
& A^{\mathrm{T}} P+P A+R_{1}-P B R_{2}^{-1} B^{\mathrm{T}} P=0 .
\end{aligned}
$$

Next we assume that the plant (4), (5) is positive real. For positive real plants a strictly positive real controller is desira.ble since the negative-feedluack closed-loop system is guaranteed to be asymptotically stable [11]. The controller obtained above, however, is not necessarily strictly positive real. For this problem, Theorem 1 of [10] can be used to obtain an $n$ th-order strictly positive real compensator $-C_{c}(s) \sim\left[\begin{array}{c|c}A_{c} & B_{c} \\ \hline-C_{c} & 0\end{array}\right]$ that minimizes the $\mathrm{II}_{2}$ performance index $J\left(A_{\mathrm{c}}, B_{\mathrm{c}}, C_{\mathrm{c}}\right)$. Since the plant $G(s) \sim\left[\begin{array}{c|c}A & B \\ \hline C & 0\end{array}\right]$ is positive real, there exists a positivedefinite matrix $Q_{0}$ satisfyiug [12]

$$
\begin{gathered}
A Q_{0}+Q_{0} A^{\mathrm{T}}=-L L^{\mathrm{T}}, \\
Q_{0} C^{\mathrm{T}}=B .
\end{gathered}
$$

As shown in [10], if the LQG weights $V_{1}, V_{2}, R_{1}, R_{2}$ are chosen according to

$$
\begin{aligned}
V_{1} & =L L^{\mathrm{T}}+B R_{2}^{-1} B^{\mathrm{T}}>0, \\
V_{2} & =R_{2}>0 \\
R_{1} & >C^{\mathrm{T}} V_{2}^{-1} C,
\end{aligned}
$$


then the dynamic compensator $-G_{c}(s)$ given by $(10),(11)$ and (12) is strictly positive real. With $-G_{c}(s)$, the negative feedback closed-loop system matrix $\bar{A}$ is now asymptotically stable as explained above.

In the following sections we consider two types of energy flow control problems in which the plant is positive real. In each case we design positive real controllers by means of the above approach.

\section{Design of an Energy Flow Controller as an Additional Interconnected Subsystem}

In this section we consider a control problem involving $r-1$ subsystems $z_{i}(s)$ interconnected by a stiffness coupling. In this problem we assume that the controller $z_{\mathrm{c}}(s)$ can interact with the subsystems only through additional coupling elements. Thus the controller can be treated as an additional $r$ th subsys. tem. The transfer functions $Z_{z}^{-1}(s)=\operatorname{diag}\left(z_{1}^{-1}(s), \ldots, z_{r-1}^{-1}(s)\right)$ and $z_{\mathrm{c}}^{-1}(s)$ are assumed to be expressed by the state space models

$$
\begin{gathered}
\dot{x}_{z}(t)=A_{z} x_{z}(t)+B_{z} u_{z}(t), \\
y_{z}(t)=C_{z} x_{z}(t), \\
\dot{x}_{\mathrm{c}}(t)=A_{\mathrm{c}} x_{c}(t)+B_{\mathrm{c}} y(t), \\
u(t)=C_{c} x_{\mathrm{c}}(t),
\end{gathered}
$$

respectively, where $x_{z}(t) \in \mathcal{R}^{n_{z}}, x_{c}(t) \in \mathcal{R}^{n_{\mathrm{c}}}, y_{z}(t) \in \mathcal{R}^{r-1}$, $u_{z}(t) \in \mathcal{R}^{r-1}$ and $y(t), u(t)$ are scalars. As shown in Fig. 4.1, $Z^{-1}(s)$ in Fig. 2.1 is now comprised of both $Z_{z}^{-1}(s)$ and $z_{\mathrm{c}}^{-1}(s)$, that is, $Z(s)=\operatorname{diag}\left[z_{1}(s), \ldots, z_{r-1}(s), z_{c}(s)\right]$, so that the total number of subsystems is $r$. Furthermore, $y_{0}(t)$ and $u_{0}(t)$ in Fig. 4.1 are given by

$$
y_{0}=\left[\begin{array}{c}
y_{z} \\
u
\end{array}\right], \quad u_{0}=\left[\begin{array}{c}
u_{z} \\
y
\end{array}\right] .
$$

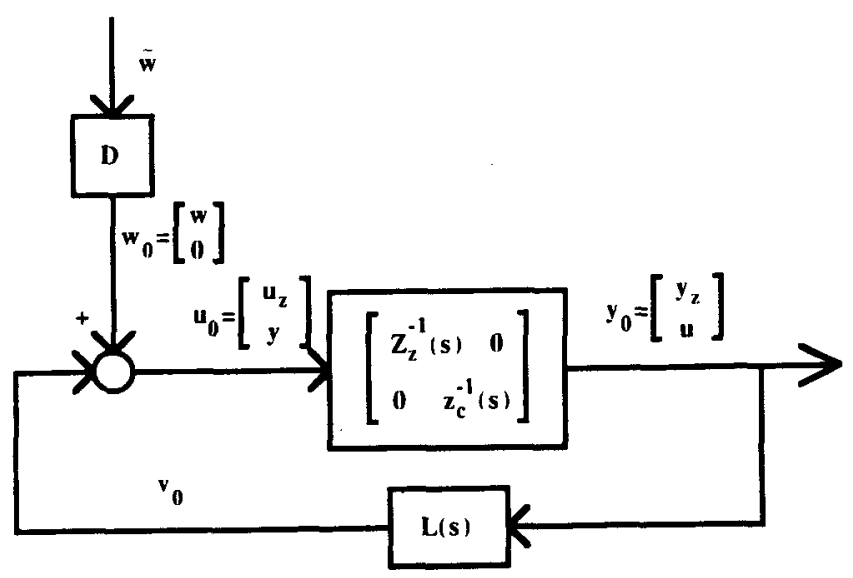

Fig. 4.1. Feedback Representation of Plant and Controller.
We now assume that the disturbance matrix $D \in \mathcal{R}^{r \times n}$ in

Fig. 2.1 is the square matrix given by

$$
D \triangleq\left[\begin{array}{cc}
D_{11} & 0 \\
0 & 0
\end{array}\right]
$$

where $D_{11} \in \mathcal{R}^{(r-1) \times(r-1)}$. This assumption for $D$ can be interpreted as that the disturbance $w_{i}(t), i=1, \ldots, r-1$, is entering $z_{i}^{-1}(s)$, whereas no disturbance enters directly into the controller $z_{\mathrm{c}}^{-1}(s)$. Thus $w_{0}(t)$ is now given by $w_{0}(t)=\left[\begin{array}{ll}w^{\mathrm{T}}(t) & 0\end{array}\right]^{\mathrm{T}}$, where $w(t) \triangleq\left[w_{1}(t) \cdots w_{r-1}(t)\right]^{\mathrm{T}}$. Since $u_{0}(t)=w_{0}(t)-v_{0}(t)$ it follows that

$$
u_{0}=\left[\begin{array}{c}
u_{z} \\
y
\end{array}\right]=\left[\begin{array}{c}
w(t) \\
0
\end{array}\right]-v_{0}(t),
$$

which implies that $u_{z}(t)$ is the force vector resulting from the difference of the disturbance forces and the coupling forces, while $y(t)$ represents the coupling force only as shown in Fig. 4.1.

After the controller is connected, the stiffness coupling $L(s)$ is given by

$$
L(s)=\frac{1}{s} C_{L}
$$

where the $r \times r$ symmetric matrix $C_{L}$ is partitioned as

$$
C_{L} \triangleq\left[\begin{array}{ll}
C_{L 11} & C_{L 12} \\
C_{L 12}^{T} & C_{L 22}
\end{array}\right]
$$

and $C_{L 11} \in \mathcal{R}^{(r-1) \times(r-1)}$

We now assume that $x_{z}$ in (20) consists of both positions and velocities so that there exists an output matrix $C_{\mathrm{p}}$ such that

$$
\int y_{z} d t=C_{\mathrm{p}} x_{z}
$$

Since $v_{0}=L(s) y_{0}=\frac{1}{s} C_{L} y_{0}$, it follows that

$$
v_{0}=C_{L} \int y_{0} d t=C_{L}\left[\begin{array}{c}
\int y_{z} d t \\
\int u d t
\end{array}\right]=C_{L}\left[\begin{array}{c}
C_{\mathrm{p}} x_{z} \\
x_{\mathrm{pc}}
\end{array}\right],
$$

where a scalar state $x_{\mathrm{pc}}(t)$ is defined by

$$
i_{\mathrm{pc}}(t) \triangleq u(t) .
$$

From (20)-(23) and (27) the feedback system in Fig. 4.1 is given by

$$
\dot{\tilde{x}}(t)=\tilde{A} \tilde{x}(t)+\tilde{D} \tilde{w}(t),
$$

where

$$
\begin{aligned}
\tilde{x}(t) \triangleq\left[\begin{array}{c}
x_{z}(t) \\
x_{\mathrm{pc}}(t) \\
x_{c}(t)
\end{array}\right], \quad \tilde{A} \triangleq\left[\begin{array}{ccc}
A_{z}-B_{z} C_{L 11} C_{\mathrm{p}} & -B_{z} C_{L 12} & 0 \\
0 & 0 & C_{\mathrm{c}} \\
-B_{\mathrm{c}} C_{L 12}^{\mathrm{T}} C_{\mathrm{p}} & -B_{\mathrm{c}} C_{L 22} & A_{\mathrm{c}}
\end{array}\right], \\
\tilde{D} \triangleq\left[\begin{array}{cc}
B_{z} D_{11} & 0 \\
0 & 0 \\
0 & 0
\end{array}\right] .
\end{aligned}
$$


We now wish to detemine $A_{c}, B_{c}$ and $C_{c}$ in (22) and (23) by means of the LQG positive real approach described in Section 3. By defining

$$
\begin{aligned}
& A \triangleq\left[\begin{array}{cc}
A_{z}-B_{z} C_{L 11} C_{\mathrm{p}} & -B_{z} C_{L 12} \\
0 & 0
\end{array}\right], \quad B \triangleq\left[\begin{array}{lll}
0 & \cdots & 1
\end{array}\right]^{\mathrm{T}}, \\
& C \triangleq\left[-C_{L 12}^{\mathrm{T}} C_{1},-C_{L 22}\right], \quad D_{1} \triangleq\left[\begin{array}{cc}
B_{z} D_{11} & 0 \\
0 & 0
\end{array}\right],
\end{aligned}
$$

then $\tilde{A}$ and $\tilde{D}$ in (29) can be written as

$$
\tilde{A}=\left[\begin{array}{cc}
A & B C_{c} \\
B_{r} \cdot C_{c} & A_{r}
\end{array}\right], \quad \tilde{D}=\left[\begin{array}{c}
D_{1} \\
0
\end{array}\right] .
$$

Thus by viewing $\left(A, B, C^{\prime}\right)$ as a realization of the plant given by (4) and (5), (29) can be interpreted as an LQG control problem. As in Section 3 we consider the performance variables

$$
z(t)=E_{1} u(t)+E_{2} u(t),
$$

where $x(t) \triangleq\left[\begin{array}{c}x_{z}(t) \\ x_{p c}(t)\end{array}\right]$ and $u(t)$ in $(23)$ is the controller ont put to be included in the cost function.

The controller is now required to minimize the energy flow into the $i$ th subsystem, that is, to minimize $P_{i}^{p}$. By defining

$$
C_{1} \triangleq\left[\begin{array}{ccc}
C_{z} & 0 & 0 \\
0 & 0 & C_{1}
\end{array}\right] \in \mathcal{R}^{r \times 2\left(n_{2}+n_{L}\right)},
$$

$P_{i}^{\mathrm{e}}$ is given by [7]

$$
P_{i}^{\mathrm{e}}=\frac{1}{2}\left[D \tilde{D}^{\mathrm{T}} C_{1}^{\mathrm{T}}\right]_{(i, i)}
$$

Thus $P_{i}^{e}$ is constant and independent of the controller gains. It thus follows from (3) that minimizing $P_{i}^{\mathrm{P}}$ is equivalent to minimizing $-P_{i}^{\mathbf{d}}$.

To express the dissipation of the $i$ th subsystem $P_{i}^{d}$ in terms of the steady state covariance $\tilde{Q} \triangleq \lim _{t \rightarrow \infty} \mathcal{E}\left[x(t) x^{\mathrm{T}}(t)\right]$, we now assume that each subsystem $z_{i}(s)$ has constant real part $c_{i}$ and define

$$
C_{\mathrm{d}} \triangleq \operatorname{diag}\left(c_{1}, \ldots, c_{r-1}, 0\right) \in \mathcal{R}^{r \times r}
$$

then $P_{i}^{\mathrm{d}}, i=1, \ldots, r-1$, can be obtained by [7]

$$
P_{i}^{\mathrm{d}}=-\left[C_{\mathrm{d}} C_{1} \tilde{Q} C_{\mathrm{I}}^{\mathrm{T}}\right]_{(i, i)},
$$

where $\tilde{Q}$ satisfies the Lyapunov equation

$$
0=\tilde{A} \tilde{Q}+\tilde{Q} \tilde{A}^{\mathbf{T}}+\tilde{D} \tilde{D}^{\mathbf{T}}
$$

Thus the cost $-P_{i}^{d}$ to be minimized is given by

$$
-P_{i}^{\mathrm{d}}=\left[C_{\mathrm{d}} C_{1} \tilde{Q} C_{1}^{\mathrm{T}}\right]_{(i, i)}
$$

Now using the definition of $\bar{Q}$ yields

$$
\begin{aligned}
-P_{i}^{\mathrm{d}} & =\left[C_{\mathrm{d}} C_{1}\left(\lim _{t \rightarrow \infty} \mathcal{E}\left[x(t) x^{\mathrm{T}}(t)\right]\right) C_{1}^{\mathrm{T}}\right]_{(i, i)} \\
& =\lim _{t \rightarrow \infty} \mathcal{E}\left[e_{i}^{\mathrm{T}} C_{\mathrm{d}} C_{1} x(t) x^{\mathrm{T}}(t) C_{1}^{\mathrm{T}} e_{i}\right] \\
& =\lim _{t \rightarrow \infty} \mathcal{E}\left[\operatorname{tr}\left[x^{\mathrm{T}}(t) C_{1}^{\mathrm{T}} e_{i} e_{i}^{\mathrm{T}} C_{\mathrm{d}} C_{1} x(t)\right]\right] \\
& =\lim _{t \rightarrow \infty} \mathcal{E}\left[x^{\mathrm{T}}(t) C_{1}^{\mathrm{T}} e_{i} c_{i} e_{i}^{\mathrm{T}} C_{1} x(t)\right] .
\end{aligned}
$$

Thus, letting the performance matrix $E_{1}$ in (30) be given by

$$
E_{1}=\sqrt{c_{i}} e_{i}^{\mathrm{T}} C_{1}
$$

corresponds to minimizing $-P_{i}^{\mathrm{d}}$.

As an illustrative numerical example we consider the three coupled oscillator system with controller as shown in Fig. 4.2, where $k_{1}=3.5, k_{2}=2.5, k_{3}=1, m_{1}=1, m_{2}=2, m_{3}=3$, $K_{12}=0.5, K_{13}=0.6, K_{23}=0.7, K_{1 c}=0.8, K_{2 c}=0.9, K_{3 c}=$ 1.0 and $c_{1}=0.1, c_{2}=0.2, c_{3}=0.3$. Furthermore, let the white noise disturbances $w_{i}(t), i=1,2,3$, have unit intensity $S_{w_{i} w_{i}}^{\prime}=1$, that is, $D=\operatorname{diag}[1,1,1,0]$. To maximize $-P_{i}^{\mathrm{P}}$, $i=1,2,3$, we set $E_{2}=0.1$ in (30). The resulting energy flow diagrams calculated by means of the steady state covariance [7] are illustrated in Fig. 4.3, where OL denotes the open-loop system and $G_{\mathrm{c} 1}, G_{\mathrm{c} 2}$ and $G_{63}$ represent the controllers designed to maximize $-P_{1}^{\mathrm{P}},-P_{2}^{\mathrm{P}}$ and $-P_{3}^{\mathrm{p}}$, respectively. Fig. 4.3 shows that the controller absorbs energy from all of the subsystems and reduces the energy dissipation from each subsystem. Furthermore, it can be seen that the controller $G_{\mathbf{c} i}$ maximizes energy flow from the $i$ th subsysten, that is, minimizes the energy dissipated by the specified subsystem.

To examine the actual recluction of vibration by these controllers, we define the steacly-state stored energy by

$$
\mathcal{E}_{i} \triangleq \frac{1}{2} m_{i} \mathcal{E}\left[x_{i}^{2}(t)\right]+\frac{1}{2} k_{i} \mathcal{E}\left[x_{i}^{2}(t)\right], \quad i=1,2,3,
$$

where $x_{i}(t)$ and $\dot{x}_{i}(t)$ are the displacement and the velocity of the $i$ th oscillator, respectively. Table 4.1 shows that each controller $G_{c i}$ successfully reduces the stored energy $\mathcal{E}_{i}$ of the corresponding $i$ th oscillator. For example, controller $G_{\mathrm{c} 1}$ reduces the stored energy of oscillator 1 to 48.32 percent of its open-loop value.

Bode gain and pliase plots of the controllers are shown in Fig. 4.4, which shows that the gain plot of controller $G_{\mathrm{cI}}$ has a peak near the coupled natural frequency of oscillator 1 , that is, $\omega_{1}=\left(k_{1}+k_{12}+k_{13}+k_{1 \mathrm{c}}\right) / m_{1}=2.3238(\mathrm{rad} / \mathrm{sec})$. Similarly, 
controller $G_{\mathrm{c} 2}$ has a gain peak near $\omega_{2}=1.516(\mathrm{rad} / \mathrm{sec})$, while controller $G_{\mathrm{c} 3}$ has a gain peak near $\omega_{3}=1.048(\mathrm{rad} / \mathrm{sec})$. These controllers are strictly positive real since their phase plots lie in the range $\left(-90^{\circ}, 90^{\circ}\right)$.

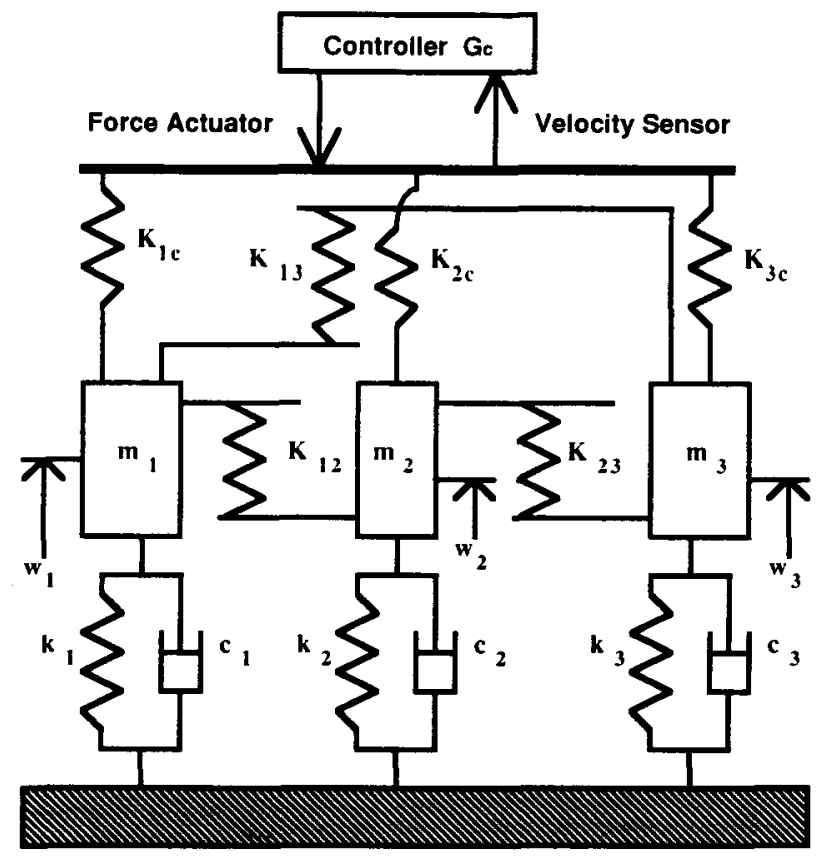

Fig. 4.2. Coupled Oscillator System with Controller.

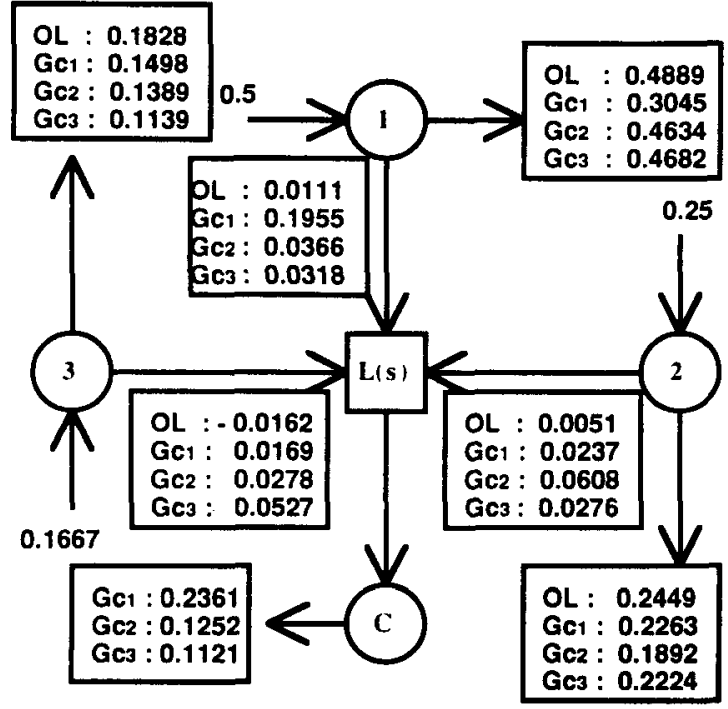

Fig. 4.3. Energy Flow among Oscillators for the Open-Lool Systen and for the Closed-Loop System with Controllers.

\begin{tabular}{|c||c||c|c|c|}
\hline Stored Energy & Open-Loop & Controller 1 & Controller 2 & Cont roller 3 \\
\hline \hline $\mathcal{E}_{1}$ & 4.2936 & 2.0747 & 3.5476 & $3 . \times 20 \%$ \\
& & $(48.32 \%)$ & $(82.63 \%)$ & $(8 \times .99 \%)$ \\
\hline $\mathcal{E}_{2}$ & 2.0556 & 1.5775 & 0.9772 & 1.6290 \\
& & $(76.74 \%)$ & $(47.54 \%)$ & $(79.25 \%)$ \\
\hline $\mathcal{E}_{3}$ & 1.3458 & 0.8542 & 0.7809 & $0.6374)$ \\
& & $(63.47 \%)$ & $(58.02 \%)$ & $(42.2 .4 \%)$ \\
\hline
\end{tabular}

Table 4.1. Stcady-State Stored Energy for Three Conpled Oscillators.
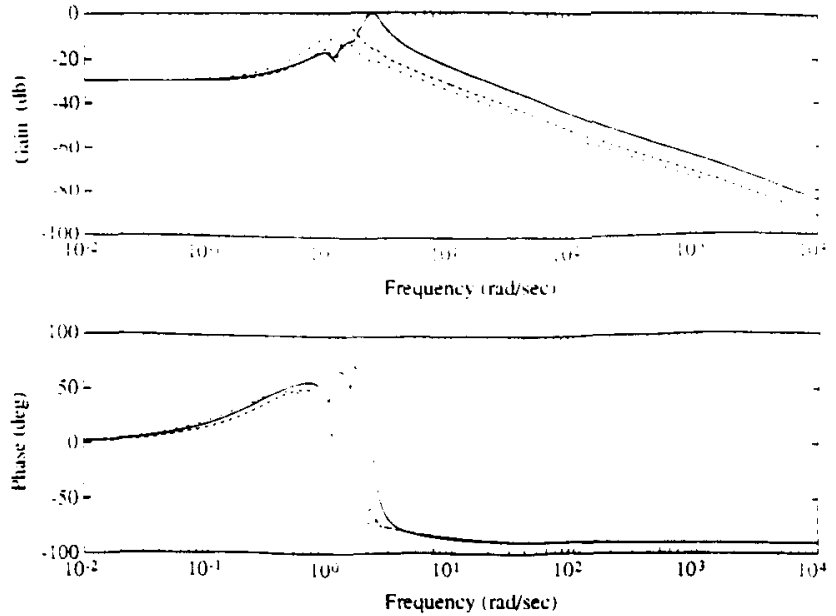

Fig. 4.4. Magnitude and Phase of Controllers $G_{c 1}$ (solid), $G_{c^{2}}$ (dlashed), $G_{c 3}($ dash-dot $)$.

5. Design of an Energy Flow Controller as a Dissipative Coupling

In the previous section, we considered the subsystem interconnection explicitly in the energy flow analysis. As an alternative approach, we view the structure as a collection of uncoupled subsystems, such as modes, which become coupled only by means of the feedback controller. In contrast to the previous section, in which the coutrol is applied to the flexible structure only through the conservative conpling, we now assume that the control force can be applied to the structure directly and design a controller to regulate energy flow among structural modes.

Consider a structure subject to unit intensity white noise disturbances $\tilde{w}_{i}(t), i=1, \ldots, n$, applied at locations $\xi_{d_{i}}$. The $i$ th actuator located at $\xi_{i}, i=1, \ldots, m$, applies a control force $u_{i}(t)$. Our goal is to design a controller that maximizes energy flow from the $i$ th structural mode. For this purpose we consider each mode as a subsystem to obtain the feedback system corresponding to Fig. 2.1 and design a dissipative controller as a dissipative coupling.

First we denote the modal decomposition of the structure by

$$
\eta(\xi, t)=\sum_{i=1}^{\infty} q_{i}(t) \psi_{i}(\xi)
$$

where $q_{i}(t)$ denotes modal coordinates and $\psi_{i}(\xi)$ denotes orthogonal eigenfunctions. Then, using the boundary conditions and orthogonality properties, it follows that the modal coordinates $q_{j}(t)$ satisfy

$\ddot{q}_{j}(t)+2 \zeta_{j} \omega_{j} q_{j}(t)+\omega_{j}^{2} q_{j}(t)=\sum_{l=1}^{m} \psi_{j}\left(\xi_{l}\right) u_{l}(t)+\sum_{l=1}^{n} \psi_{j}\left(\xi_{\mathrm{d} l}\right) \tilde{w}_{l}(t)$, 
where we assume proportional damping $2 \zeta_{j} \omega_{j}$. From $(38), \dot{\eta}\left(\xi_{i}, t\right)$ is the velocity of the structure at the $i$ th actuator position $\xi_{i}$ and we assume that $m$ velocity sensors are also located at these positions. Hence the sensors and actuators are colocated and dual.

Now we consider $r$ structural modes and define

$$
\begin{aligned}
x(t) & \triangleq\left[q_{1}(t) \dot{q}_{1}(t) q_{2}(t) \dot{q}_{2}(t) \cdots q_{r}(t) \dot{q}_{r}(t)\right]^{\mathrm{T}}, \\
u(t) & \triangleq\left[u_{1}(t) u_{2}(t) \cdots u_{m}(t)\right]^{\mathrm{T}}, \\
\tilde{w}(t) & \triangleq\left[\tilde{w}_{1}(t) \tilde{w}_{2}(t) \cdots \tilde{w}_{n}(t)\right]^{\mathrm{T}} \\
y(t) & \triangleq\left[\dot{\eta}\left(\xi_{1}, t\right) \dot{\eta}\left(\xi_{2}, t\right) \cdots \dot{\eta}\left(\xi_{r}, t\right)\right]^{\mathrm{T}} .
\end{aligned}
$$

Then from (38) we obtain the state space model

$$
\begin{gathered}
\dot{x}(t)=A x(t)+B u(t)+D \tilde{w}(t), \\
y(t)=B^{\mathrm{T}} x(t),
\end{gathered}
$$

where

$$
\begin{gathered}
\left.\left.A \triangleq \begin{array}{c}
\text { block }-\operatorname{diag} \\
i=1, \ldots, r
\end{array}\right] \begin{array}{cc}
0 & 1 \\
-\omega_{i}^{2} & -2 \zeta_{i} \omega_{i}
\end{array}\right] \in \mathcal{R}^{2 r \times 2 r}, \\
B \triangleq\left[\begin{array}{ccc}
0 & \ldots & 0 \\
\psi_{1}\left(\xi_{1}\right) & \ldots & \psi_{1}\left(\xi_{m}\right) \\
0 & \ldots & 0 \\
\psi_{2}\left(\xi_{1}\right) & \ldots & \psi_{2}\left(\xi_{m}\right) \\
\vdots & : & \vdots \\
\vdots & : & : \\
0 & \ldots & 0 \\
\psi_{r}\left(\xi_{1}\right) & \ldots & \psi_{r}\left(\xi_{m}\right)
\end{array}\right], D \triangleq\left[\begin{array}{ccc}
0 & \ldots & 0 \\
\psi_{1}\left(\xi_{\mathrm{d}_{1}}\right) & \ldots & \psi_{1}\left(\xi_{\mathrm{d}_{n}}\right) \\
0 & \ldots & 0 \\
\psi_{2}\left(\xi_{\mathrm{d}_{1}}\right) & \ldots & \psi_{2}\left(\xi_{\mathrm{d}_{n}}\right) \\
\vdots & : & \vdots \\
: & : & : \\
0 & \ldots & 0 \\
\psi_{r}\left(\xi_{\mathrm{d}_{1}}\right) & \ldots & \psi_{r}\left(\xi_{\mathrm{r}_{n}}\right)
\end{array}\right],
\end{gathered}
$$
and $B \in \mathcal{R}^{2 \tau \times m}$ and $D \in \mathcal{R}^{2 \tau \times \pi}$.

To obtain the feedback system equivalent to Fig. 2.1 we introduce the diagonal matrix $B_{0}$ defined by

$$
B_{0} \triangleq \operatorname{diag}(0,1,0,1, \ldots, 0,1) \in \mathcal{R}^{2 \tau \times 2 r},
$$

and define

$$
\begin{gathered}
Z^{-1}(s) \sim\left[\begin{array}{c|c}
A & B_{0} \\
\hline B_{0} & 0
\end{array}\right], \\
y_{0}(t) \triangleq B_{0} x(t) \in \mathcal{R}^{2 r}, \\
w_{0}(t) \triangleq D \tilde{w}(t) \in \mathcal{R}^{2 r}, \\
v_{0}(t) \triangleq-B u(t) \in \mathcal{R}^{2 r} .
\end{gathered}
$$

We thus obtain Fig. 5.1 where the coupling $L(s)$ is defined by

$$
L(s) \triangleq-B G_{\mathrm{c}}(s) B^{\mathrm{T}}
$$

Now using the LQG positive real approach we design a strictly positive real controller $G_{\mathrm{c}}(s)$ satisfying

$$
G_{c}(s)+G_{c}^{*}(s)<0, \operatorname{Re}[s]>0,
$$

$$
\begin{aligned}
L(s)+L^{*}(s) & =-B G_{\mathrm{c}}(s) B^{\mathrm{T}}-\left[B G_{\mathrm{c}}(s) B^{\mathrm{T}}\right]^{*} \\
& =-B\left[G_{c}(s)+G_{\mathrm{c}}^{*}(s)\right] B^{\mathrm{T}} \\
& \geq 0
\end{aligned}
$$

for $\operatorname{Re}[s]>0$. Thus the coupling $L(s)$ serves as a dissipative controller which controls the energy flow among the structural modes. Our goal is to design $G_{\mathrm{c}}(s)$ so that $L(s)$ maximizes energy flow from a specified mode.

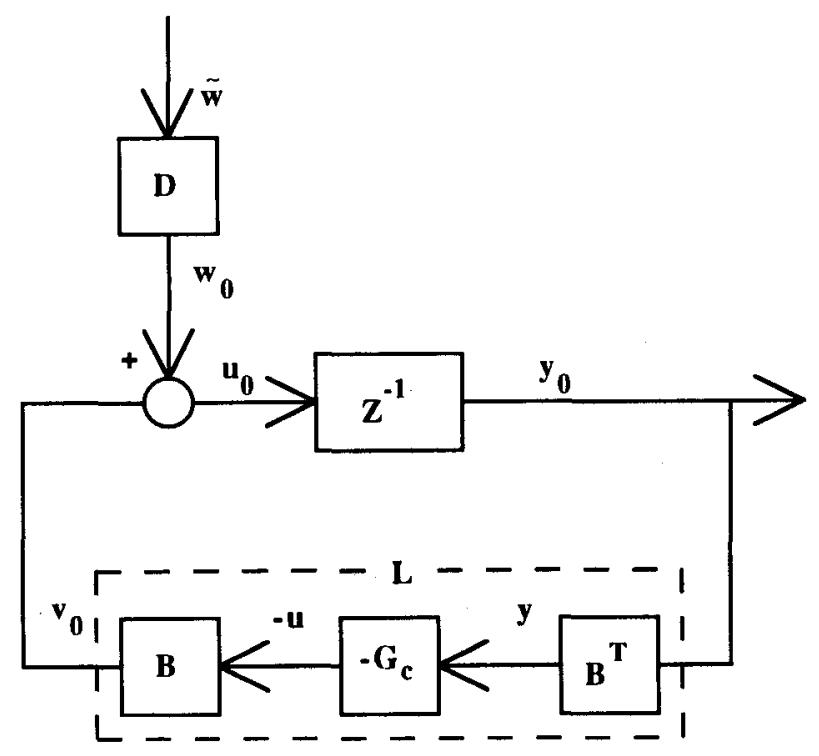

Fig. 5.1. Feedback Representation of Coupled Structural Modes.

Next we consider a realization of the feedback system in Fig. 5.1. The transfer functions $Z^{-1}(s)$ and $G_{c}(s)$ are expressed by the state space models

$$
\begin{gathered}
\dot{x}(t)=A x(t)+B_{0} u_{0}(t), \\
y_{0}(t)=B_{0} x(t), \\
\dot{x}_{\mathrm{c}}(t)=A_{c} x_{\mathrm{c}}(t)+B_{\mathrm{c}} y(t), \\
u(t)=C_{\mathrm{c}} x_{\mathrm{c}}(t),
\end{gathered}
$$

respectively. Since $u_{0}=w_{0}-v_{0}$ and $B_{0} B=B$, it follows from $(40),(41)(49)$ - (52) that

$$
\begin{gathered}
\dot{x}(t)=A x(t)+B C_{\mathrm{c}} x_{\mathrm{c}}(t)+B_{0} w_{0}(t), \\
\dot{x}_{\mathrm{c}}(t)=A_{\mathrm{c}} x_{\mathrm{c}}(t)+B_{\mathrm{c}} B^{\mathrm{T}} x(t) .
\end{gathered}
$$

Thus the feedback system (53) and (54) is given by

$$
\dot{\tilde{x}}(t)=\tilde{A} \tilde{x}(t)+\tilde{D} \tilde{w}(t)
$$

so that $L(s)$ satisfies 
where

$$
\begin{gathered}
\tilde{x}(t) \triangleq\left[\begin{array}{c}
x(t) \\
x_{\mathrm{c}}(t)
\end{array}\right] \in \mathcal{R}^{4 r}, \quad \tilde{A} \triangleq\left[\begin{array}{cc}
A & B C_{\mathrm{c}} \\
B_{\mathrm{c}} B^{\mathrm{T}} & A_{\mathrm{c}}
\end{array}\right] \in \mathcal{R}^{4 r \times 4 r}, \\
\tilde{D} \triangleq\left[\begin{array}{c}
B_{0} D \\
0
\end{array}\right] \in \mathcal{R}^{4 r \times 2 r}
\end{gathered}
$$

By setting $C=B^{\mathrm{T}}$ in (5), it can be seen that $\tilde{A}$ has the usual closed-loop structure.

Now we choose the performance varialsle in (30) to maximize energy flow from the ith structural mode, that is, to maximize $-P_{i}^{\text {p }}$. By the same argunent as in the previous section, this is equivalent to mininizing $-P_{i}^{\mathrm{rl}}$. By defining

$$
C_{1 / 2} \triangleq\left[B_{0} \quad 0_{2 r \times 2 r}\right] \in \mathcal{R}^{2 r \times 4 r}
$$

and

$$
C_{\mathrm{da}} \triangleq \operatorname{diag}\left(0,2 \zeta_{1} \omega_{1}, 0,2 \zeta_{2} \omega_{2}, \ldots, 0,2 \zeta_{r} \omega_{r}\right)
$$

then $P_{i}^{\mathrm{d}}, i=1, \ldots, r$, is given loy

$$
P_{i}^{\mathrm{d}}=-\left[C_{1 i} C_{1 \alpha}^{\prime} \bar{Q} C_{1 a}^{\prime T}\right]_{(2 i, 2 i)},
$$

where $\bar{Q}$ satisfies the Lyapmuov equation

$$
0=\tilde{A} \tilde{Q}+\tilde{Q} \tilde{A}^{\mathrm{T}}+\tilde{D} \tilde{D}^{\mathrm{T}}
$$

Thus the performance index to be minimized is given by

$$
\left[C_{\mathrm{da}} C_{12} \bar{Q} C_{1+1}^{\mathrm{T}}\right]_{(2,2 i)}
$$

and in the similar manneras rhe previous section the performance matrix $E_{1}$ in (30) is given by

$$
E_{1}=\sqrt{2 \zeta_{i} \omega_{i}} e_{i}^{\mathrm{T}} C_{1 a}
$$

Finally, since (40) and (41) comprise a state space model of the structure given by (39), it follows that the plant $(A, B, C)$ is strictly positive real. We can thus obtain a strictly positive real controller $-G_{c}(s)$ in the same manner as in the previons section.
As a numerical example, we now consider the simply supported uniform Bermoulli-Euler beam of length $L$ in Fig. 5.2. The partial differential equation for the transverse deflection $\eta(\xi, t)$ is given by

$\rho \frac{\partial^{2} \eta(\xi, t)}{\partial t^{2}}+\frac{\partial^{2}}{\partial \xi^{2}}\left[E I_{\mathrm{A}} \frac{\partial^{2} \eta(\xi, t)}{\partial \xi^{2}}\right]=\sum_{i=1}^{r} \delta\left(\xi-\xi_{i}\right) u_{i}(t)+\sum_{i=1}^{n} \delta\left(\xi-\xi_{\mathrm{d}_{i}}\right) \tilde{r}_{i}(t)$.

with boundary conditions

$$
\left.\eta(\xi, t)\right|_{\xi=0, L}=0 .\left.\quad E I_{\mathrm{A}} \frac{\partial^{2} \eta(\xi, t)}{\partial \xi^{2}}\right|_{\xi=0 . L}=0,
$$

where $\rho$ is the mass per unit length, $\bar{c}$ is the damping per mit length of the $i$ th mode, $E I_{A}$ is the bending stiffness.

By substituting (38) into (61) and using the orthogonality properties

$$
\int_{0}^{L} \rho \psi_{i}(\xi) \psi_{j}(\xi) d \xi=\delta_{i j}, \quad \int_{0}^{L} E I_{\lambda} \frac{\partial^{4}}{\partial \xi^{4}} l^{\prime}{ }_{i}(\xi) \psi_{j}(\xi) d \xi=\omega_{i}^{2} \delta_{i j}
$$

where $\delta_{i j}$ is the Kronecker clelta, we obtain (39) with natural frequencies $\omega_{i}$ and eigenfunctions $\psi_{i}(\xi)$ given by

$$
\omega_{i}=\sqrt{\frac{E I_{\mathrm{A}}}{\rho}}\left(\frac{i \pi}{L}\right)^{2}, \quad \varphi_{i}(\xi)=\sqrt{\frac{2}{\rho L}} \sin \frac{i \pi \xi}{L}, \quad i=1,2,3 \ldots
$$

For numerical simplicity, let $L=\pi$ and $E I_{\mathrm{A}}=\rho=\frac{2}{\pi}$ so that

$$
\omega_{i}=i^{2}, \quad \psi_{i}(\xi)=\sin i \xi, \quad i=1,2,3, \ldots
$$

Furthermore, two actuators are assumed to be located at $\xi_{1}=$ $1, \xi_{2}=2$, and a white noise disturbance with unit intensity is entering at $\xi_{d_{1}}=1.7$. Finally, we set $\zeta_{1}=\zeta_{2}=\zeta_{3}=0.01$ and $E_{2}=I$ in (30) and retain the first three modes. The resulting energy flows are shown in Fig. 5.3 for controllers $G_{\mathrm{c} 1}, G_{\mathrm{c} 2}$ and $G_{c 3}$ designed to maximize $-P_{1}^{\mathrm{p}},-P_{2}^{\mathrm{p}}$ and $-P_{3}^{\mathrm{p}}$, respectively. These results show that each controller maximizes the energy flow from a specified mode and that the energy removed from each subsystem is dissipated by the coupling.

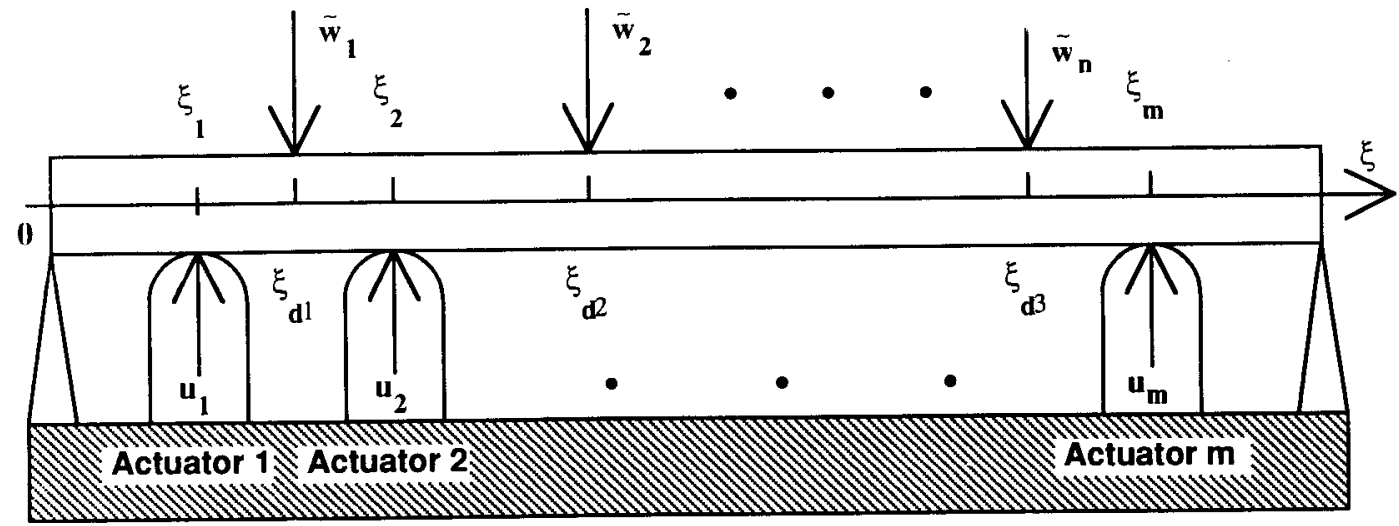

Fig. 5.2. Simply Supported Uniform Beam. 


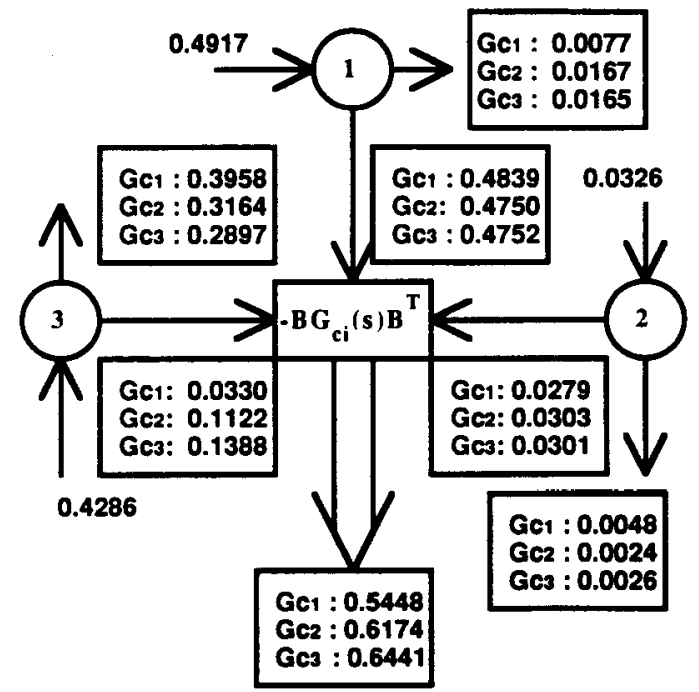

Fig. 5.3. Energy Flow among Structural Modes with Controllers.

Now we define the stearly-state modal energy by

$$
\mathcal{E}_{i} \triangleq \frac{1}{2} \mathcal{E}\left[\dot{\Psi}_{i}^{2}(t)\right]+\frac{1}{2} \omega_{i}^{2} \mathcal{E}\left[q_{i}^{2}(t)\right], \quad i=1,2,3
$$

and the result is shown in Table 5.1. Table 5.1 shows that controller $G_{c i}$ successfully reduces the stored energy $\mathcal{E}_{i}$ of the $i$ th mode.

\begin{tabular}{|c||c||c|c|c|}
\hline Modal Energy & Open-Loop & Controller 1 & Controller 2 & Cont roller 3 \\
\hline \hline $\mathcal{E}_{1}$ & 24.5847 & 0.3873 & 0.8288 & 0.8276 \\
& & $(1.58 \%)$ & $(3.37 \%)$ & $(3.37 \%)$ \\
\hline $\mathcal{E}_{2}$ & 0.8160 & 0.0606 & 0.0295 & 0.0482 \\
& & $(7.43 \%)$ & $(3.17 \%)$ & $(5.91 \%)$ \\
\hline $\mathcal{E}_{3}$ & 4.7618 & 2.1960 & 1.7561 & 1.3266 \\
& & $(46.12 \%)$ & $(36.88 \%)$ & $(27.86 \%)$ \\
\hline
\end{tabular}

Table 5.1. Steady-State Modal Energy of the ith Mode of a Flexible Beam.

\section{Design of an Energy Flow Controller for Relative Force}

As a further illustration of the approach of the previous section, we consider the interconnection of two positive real systems $z_{i}(s), i=1,2$, by means of a relative force controller. The controller thus serves as a dissipative coupling as in the previous section. This controller can be viewed as a device for regulating energy flow between two nominally uncoupled subsystems or as an interstitial device a.ttached to two points on a single structure.

Let $Z^{-1}(s)$ and $G_{c}(s)$ represent the transfer functions of the two uncoupled strictly positive real systems and the controller, respectively, and assume these systems have the state space realizations

$$
\dot{x}_{\mathrm{p}}(t)=A x_{\mathrm{p}}(t)+B_{\mathrm{p}} u_{0}(t),
$$

$$
\begin{gathered}
y_{0}(t)=C_{\mathrm{p}} x_{\mathrm{p}}(t), \\
\dot{x}_{\mathrm{c}}(t)=A_{c} x_{\mathrm{c}}(t)+B_{\mathrm{c}} y(t), \\
u(t)=C_{\mathrm{c}} x_{c}(t),
\end{gathered}
$$

respectively, where $x_{\mathrm{p}}(t) \in \mathcal{R}^{n}, u(t) \in \mathcal{R}^{2}, x_{\mathrm{c}}(t) \in \mathcal{R}^{n}$. Now $y_{0}(t) \in \mathcal{R}^{2}$ is the velocity vector of the two uncoupled systems and the scalars $y(t)$ and $u(t)$ represent the relative velocity and relative force, respectively.

To obtain the relative velocity $y(t)$ and the coupling force $v_{0}(t) \in \mathcal{R}^{2}$ we define $\tilde{B}$ as

$$
\tilde{B} \triangleq\left[\begin{array}{c}
1 \\
-1
\end{array}\right],
$$

so that $y(t)=\tilde{B} y_{0}(t)$ and $v_{0}(t)=-\tilde{B} u(t)$. With $\tilde{B}$ given by (67), the feedback system shown in Fig. 6.1 is equivalent to Fig. 2.1, where in Fig. 6.1, $L(s)$ is given by

$$
L(s) \triangleq-\bar{B} G_{\mathrm{c}}(s) \tilde{B}^{\mathrm{T}}
$$

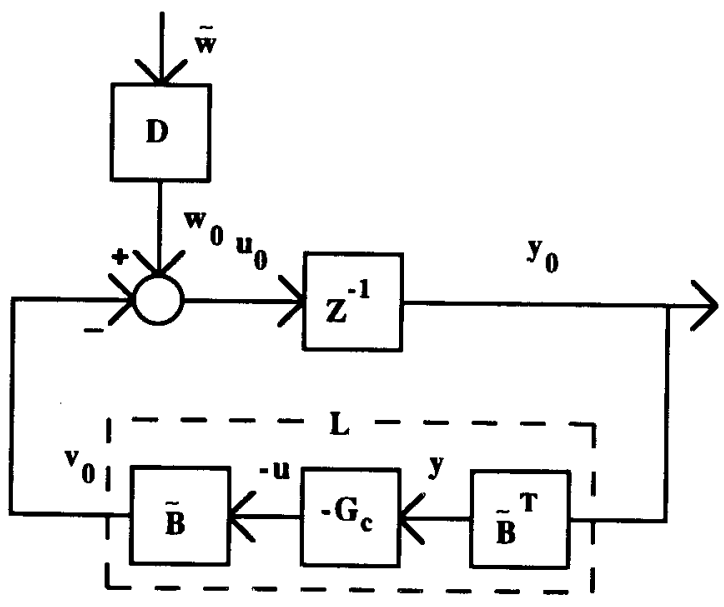

Fig. 6.1. Feedback Representation of Coupled System.

Thus the coupling $L(s)$ serves as a dissipative controller which controls energy flow between the subsystems.

Now (65) and (66) can be rewritten with $\tilde{B}$ as

$$
\begin{gathered}
\dot{x}_{\mathrm{c}}(t)=A_{c} x_{c}(t)+B_{c} \tilde{B}^{\mathrm{T}} y_{0}(t), \\
v_{0}(t)=-\tilde{B} C_{\mathrm{c}} x_{\mathrm{c}}(t) .
\end{gathered}
$$

Thus the feedback system $(63),(64),(69)$ and $(70)$ is given by

where

$$
\dot{\tilde{x}}(t)=\tilde{A} \tilde{x}(t)+\tilde{D} \tilde{w}(t),
$$

$$
\begin{gathered}
\tilde{x}(t) \triangleq\left[\begin{array}{l}
x_{\mathrm{p}}(t) \\
x_{\mathrm{c}}(t)
\end{array}\right] \in \mathcal{R}^{2 n}, \tilde{A} \triangleq\left[\begin{array}{cc}
A & B_{\mathrm{p}} \tilde{B} C_{\mathrm{c}} \\
B_{\mathrm{c}} \tilde{B}^{\mathrm{T}} C_{\mathrm{p}} & A_{\mathrm{c}}
\end{array}\right] \in \mathcal{R}^{2 n \times 2 n}, \\
\tilde{D} \triangleq\left[\begin{array}{c}
B_{\mathrm{p}} D \\
0
\end{array}\right] \in \mathcal{R}^{2 n \times 2} .
\end{gathered}
$$


By setting $B=B_{\mathrm{p}} \tilde{B}$ and $C=\tilde{B}^{\mathrm{T}} C_{\mathrm{p}}, \tilde{A}$ can be written as $\tilde{A}=$ $\left[\begin{array}{cc}A & B C_{\mathrm{c}} \\ B_{\mathrm{c}} C & A_{\mathrm{c}}\end{array}\right]$ so that (71) has the usual closed-loop structure. Now we choose the performance variable $E_{1} x(t)$ to maximize the energy flow from the $i$ th subsystem, where $i=1,2$. By the same argument in the previous sections this is equivalent to minimizing $-P_{i}^{\mathrm{d}}$.

Define the $2 \times 2$ damping matrix $C_{\mathrm{d} 1}$ by

$$
C_{\mathrm{d} 1} \triangleq \operatorname{diag}\left[c_{1}, c_{2}\right]
$$

where $c_{i} \triangleq \operatorname{Re}\left[z_{i}(s)\right], i=1,2$. Then $P_{i}^{\mathrm{d}}, i=1,2$, is given by

$$
P_{i}^{\mathrm{d}}=-\left[C_{\mathrm{d} 1} C_{\mathrm{pa}} \tilde{Q} C_{\mathrm{pa}}^{\mathrm{T}}\right]_{(i, i)}
$$

where $C_{\mathrm{pa}} \triangleq\left[\begin{array}{ll}C_{\mathrm{p}} & 0\end{array}\right] \in \mathcal{R}^{2 \times 2 n}$, and $\bar{Q}$ satisfies the Lyapunov equation

$$
0=\bar{A} \bar{Q}+\tilde{Q} \tilde{A}^{\mathrm{T}}+\tilde{D} \tilde{D}^{\mathrm{T}}
$$

Thus the performance matrix $E_{1}$ in $(30)$ is given by

$$
E_{1}=\sqrt{c_{i}} e_{i}^{\mathrm{T}} C_{\mathrm{pa}}
$$

Since the plant represented by $(A, B, C)$ is strictly positive real, we can use the positive real control approach to obtain the strictly positive real controller $-G_{\mathrm{c}}(s)$.

To illustrate this approach we consider the two oscillator system with the coupling $L(s)$ shown in Fig. 6.2, where $f$ represents the relative force. For illustrative purposes we set $k_{1}=$ $10, k_{2}=2, m_{1}=0.3, m_{2}=0.4$, and $c_{1}=0.1, c_{2}=0.2$, and let the white noise disturbances $w_{i}(t), i=1,2$, have unit intensity, that is, $D=I$. By setting $E_{2}=0.1$ in (30) we design the controllers $G_{\mathrm{c} 1}$ and $G_{\mathrm{c} 2}$ to maximize $-P_{1}^{\mathrm{p}}$ and $-P_{2}^{\mathrm{p}}$, respectively. The resulting energy flows shown in Fig. 6.3 show that each controller successfully removes energy from the specified subsystem by minimizing the dissipated energy flow out of the subsystem. The steady-state stored energy $\mathcal{E}_{i}, i=1,2$, defined by (37) is listed in Table 6.1, which shows that each controller successfully reduces the stored energy of the corresponding oscillator. Furthermore, the Bode plots of the controllers in Fig. 6.4 show that the controllers are strictly positive real.

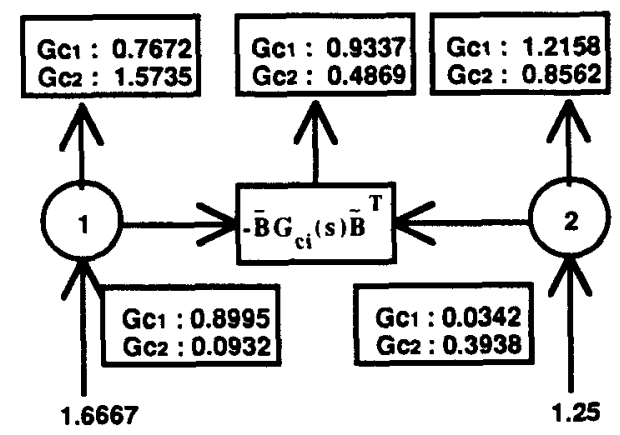

Fig. 6.3. Energy Flow between Oscillators with Controllers.

\begin{tabular}{|c||c|c|c|}
\hline Stored Energy & Open-Loop & Cont roller 1 & Cont roller 2 \\
\hline \hline $\mathcal{E}_{1}$ & 5.0 & 2.6401 & 4.5683 \\
& & $(52.08 \%)$ & $(91.37 \%)$ \\
\hline $\mathcal{E}_{2}$ & 2.5 & 2.3549 & 1.2041 \\
& & $(94.20 \%)$ & $(48.16 \%)$ \\
\hline
\end{tabular}

Table 6.1. Steady-State Stored Energy for Two Coupled Oscillators with Relative Force Actuator.
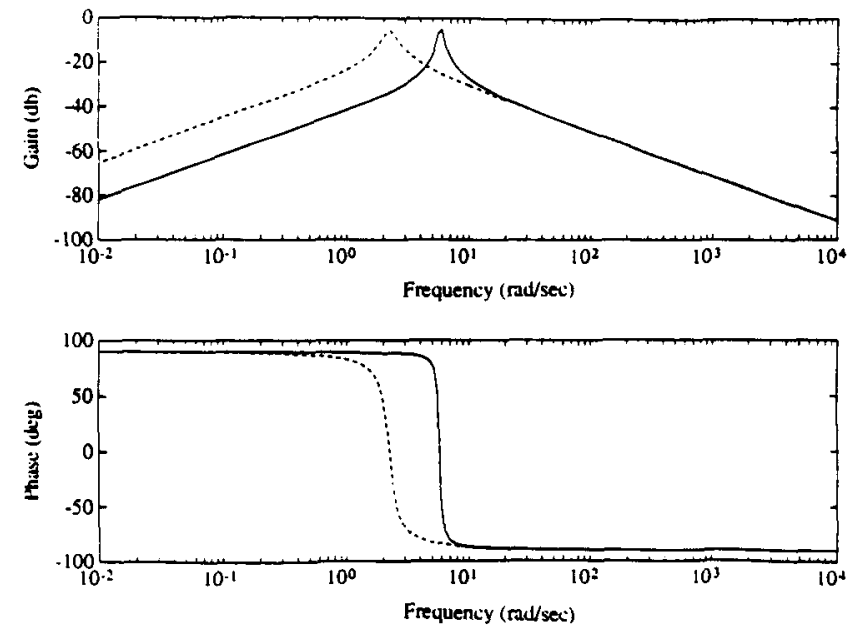

Fig. 6.4. Magnitude and Phase of Controllers $G_{\mathrm{c} 1}\left(\right.$ solirl) and $G_{\mathrm{c} 2}$ (dashed).

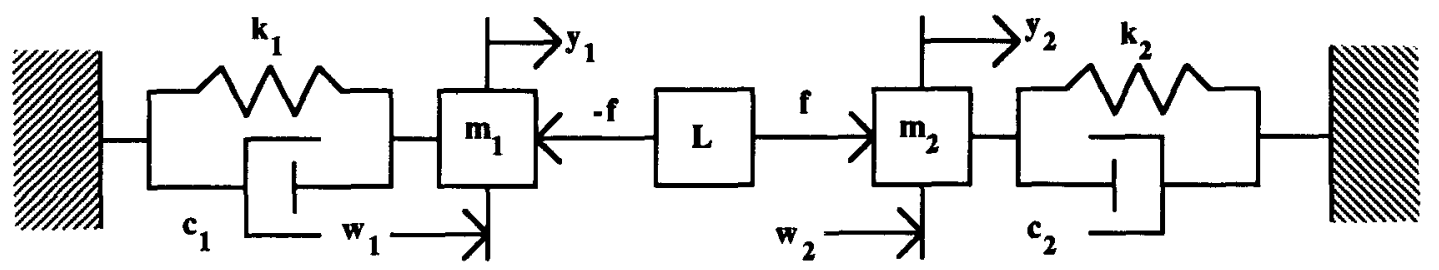

Fig. 6.2. Two Oscillator System with Relative Force Controller Coupling. 
In this paper we applied energy flow morlels obtained in [7]-[9] to design energy flow controllers for modal subsystems. By using the LQG positive real control approach, each controller was considered as either an additional subsystem or as a dissipative coupling. Each resulting controller was shown to maxinize energy flow from the specified subsystem. Furthermore, closed-loop asymptotic stability is guaranteed since strictly positive real controllers were designed in a negative feedback loop. These features were demonstrated by mmerical examples.

Acknowledgments: This research was supported in part by the Air Force Office of Scientific Research under Girant F496i2092-J-0127 and the NASA SERC Grant NAGW-1335.

\section{References}

[1] Mace, B. R., "Active Control of Frexural Vibrations," J. Sound Vib., Vol. 114, pp. 253-270, 1987.

[2] Von Flotow, A. H., and Shäfer, B. "Wave-Absorbing Controllers for a Flexible Beam," J. Guidl. Contr. Dyn., Vol. 9 , pp. $673-680,1986$.

[3] Miller, D. W., Hall, S. R., and von Flotow, A. H., "Optimal Control of Power Flow at Structural Junctions," J. Sound Vil., Vol. 140, No. 3, p1. 475-497, 1990.

[4] Pan, J., and Hansen, C. H., "Active Control of Total Vibratory Power Flow in a Beam. I: Physical System Analysis," J. Acoust. Soc. Amer., Vol. 89, pp. 200-209, 1991.

[5] MacMartin, D. G., and Hall, S. R., "Control of Uncertain Structures Using an $H_{\infty}$ Power Flow Approach," J. Guid. Contr. Dyn., Vol. 14, pp. 521-530, 1991.

[6] MacMartin, D. G., and Hall, S. R., "Broadband Control of Flexible Structures using Statistical Energy Analysis Concepts," submitted to .J. Ciuid. Contr. Dyn.

[7] Kishimoto, Y., and Bernstein, D. S., "Thermodynamic Modeling of Interconnected Systems: I. Conservative Coupling," submitted to J. Sound Vib.

[8] Kishimoto, Y., and Bernstein, D. S., "Thermodynamic Modeling of Interconnected Systems: II. Dissipative Coupling," submitted to J. Sound Vib.

[9] Wyatt, J. L., Siebert, W. M., and Tan, H. N., "A Frequency Domain Inequality for Stochastic Power Flow in Linear Networks," IEEE Trans. Circ. Sys., Vol. 31, pp. 809-814, 1984.

[10] Lozano-Leal, R. and Joshi, S. M., "On the Design of Dissipative LQG-type Controllers," Proc. IEEE Conf. Der. Contr., Honolulu, HI, pp. 3492-3495, 1990.

[11] Benhabib, R. J., Iwens, R. P., and Jackson, R. L., "Stability of Large Structure Control Systems using Positivity Concepts," J. Guid. Contr., Vol. 4, pp. 47-494, 1981.

[12] Anderson, B. D. O. and Vongpanitlerd, S., Network Analysis and Synthesis: A Modern Systems Theory Approach, Englewood Cliffs, NJ, Prentice-Hall, 1973. 